\title{
Background of SAM Atom-Fraction Profiles
}

\author{
Author ReMOVED FOR DOUbLe-Blind ReVIEWING \\ Affiliation Removed for Double-Blind Reviewing \\ (January 26, 2017)
}

\begin{abstract}
Atom-fraction profiles acquired by SAM (scanning Auger microprobe) have important applications, e. g. in the context of alloy surface engineering by infusion of carbon or nitrogen through the alloy surface. However, such profiles often exhibit an artifact in form of a background with a level that anti-correlates with the local atom fraction. This article presents a theory explaining this phenomenon as a consequence of the way in which random noise in the spectrum propagates into the discretized differentiated spectrum that is used for quantification. The resulting model of "energy channel statistics" leads to a useful semi-quantitative background reduction procedure, which is validated by applying it to simulated data. Subsequently, the procedure is applied to an example of experimental SAM data. The analysis leads to conclusions regarding optimum experimental acquisition conditions. The proposed method of background reduction is based on general principles and should be useful for a broad variety of applications.
\end{abstract}

\section{INTRODUCTION}

The mathematical symbols used in this article are compiled in Table I.

A SAM (scanning Auger microprobe) can be conceived as a scanning electron microscope that operates in UHV (ultra-high vacuum) and is equipped with an energy analyzer for Auger electrons, ${ }^{1,2}$ thus AES (Auger electron spectrometry). ${ }^{3}$ Previous work of our group extensively employed SAM to determine AFDPs (atom-fraction-depth profiles) of carbon and nitrogen in structural alloys engineered by infusing these elements though the alloy surface. ${ }^{4-7}$ Driving the process at sufficiently low temperature generates a uniform, precipitate-free "case" (hard shell) with a thickness of typically $25 \mu \mathrm{m}$ and maximum concentrations of interstitially dissolved carbon or nitrogen that corresponding to $\approx 10^{5}$ times the equilibrium solubility. Such SECIS (surface engineering by concentrated interstitial solute - carbon, nitrogen) is of great technological importance as it enables immense improvements of mechanical properties and corrosion resistance. The method we have established for determining the atomfraction-depth profile $X[z]$ of carbon or nitrogen is illustrated in Fig 1. (As infusion of interstitial solute expands the lattice parameter of the alloy matrix, ${ }^{8}$ the solute atom fraction $X$ is a more useful parameter here than the concentration.) The method consists of (i) preparing a polished cross-section vertical to the alloy surface and (ii) SAM spot measurements on this cross section at equally spaced locations along a

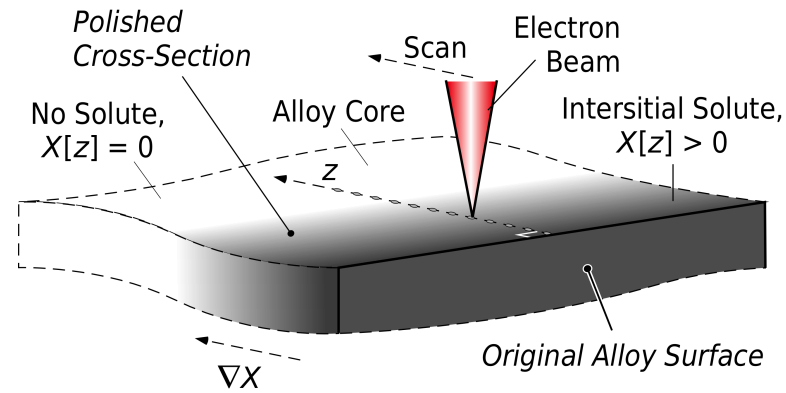

FIG. 1: Experimental setup for obtaining the AFDP (atomfraction-depth profile) $X^{\mathrm{xp}}[z]$ of interstitial solute in an alloy specimen treated by SECIS (surface engineering by concentrated interstitial solute - carbon, nitrogen).

straight line vertical to the alloy surface, i. e. a linescan.

For carbon, Fig 2 presents an an experimentally acquired AFDP ${ }^{{ }^{x p}} X[z]$ in AISI-316L (austenitic stainless steel). This material was SECIS-engineerid by lowtemperature carburization from a gas phase. The profile was measured with a PHI-680 SAM (PHI, Chanhassen, MN, USA), operating with a field-emission gun based on a Schottky emitter, a secondary electron detector, and an axial cylindrical-mirror analyzer with a multichannel detector to collect Auger electrons produced during electron imaging. Further, the instrument is equipped for sputtering the specimen surface with an inert gas (Ar) using a PHI 06-350 ion gun (PHI). For recording AFDPs, the electron probe can be positioned automatically along a straight scan line. In order to minimize the error in absolute distances between the scan points, the system has been carefully 


\section{TABLE I: Symbol Definitions}

\begin{tabular}{|c|c|}
\hline$E$ & Energy. \\
\hline$E_{n}$ & Center energy of detector channel $n$. \\
\hline$I[E]$ & $\begin{array}{l}\text { Spectral "intensity" (spectral density of Auger electron } \\
\text { current). }\end{array}$ \\
\hline$I_{n}$ & Auger electron current in energy channel $n$. \\
\hline$J[E]$ & $J[E]:=I^{\prime}[E] \equiv \mathrm{d} I / \mathrm{d} E$ \\
\hline$J_{n}$ & $J\left[E_{n}\right]$ \\
\hline$\Delta I[E]$ & Random fluctuation added to $I[E]$. \\
\hline$\Delta I_{n}$ & $\Delta I\left[E_{n}\right]$ \\
\hline$\sigma_{\mathrm{I}}$ & Standard deviation of fluctuations $\Delta I$. \\
\hline$X$ & Atom fraction of interstitial solute (carbon or nitrogen). \\
\hline$X^{\mathrm{M}}$ & Atom fraction of metal atoms. \\
\hline$z$ & Linescan coordinate; depth below specimen surface. \\
\hline$z_{m}$ & Location of point $m$ along the scan line. \\
\hline$X[z]$ & $\begin{array}{l}\text { Fraction-depth profile - atom fraction (concentration) as a } \\
\text { function of depth } z \text {. }\end{array}$ \\
\hline$X_{m}$ & $\equiv X\left[z_{m}\right]$, discretized fraction-depth profile. \\
\hline th $X[z]$ & Theoretical fraction-depth profile. \\
\hline $\mathrm{xp}_{X}[z]$ & Experimental fraction-depth profile. \\
\hline${ }^{n r} X[z]$ & Noise-reduced $X_{\mathrm{xp}}[z]$ \\
\hline $\operatorname{bg}_{X}[z]$ & Background of fraction-depth profile. \\
\hline$\alpha, \beta$ & Sensitivity factors. \\
\hline$\sigma_{\mathrm{E}}$ & Standard deviation of Gauss function-like peak in $I[E]$. \\
\hline$J^{\oplus}, J^{\ominus}$ & Maximum and minimum $J$ \\
\hline$S$ & $\left(J^{\oplus}-J^{\ominus}\right)$ \\
\hline$S_{m}$ & $\equiv S\left[z_{m}\right]$ \\
\hline${ }^{\circ} S$ & Absolute sensitivity factor. \\
\hline$\langle S\rangle_{m}$ & Mean value of $S$ over a continuous range of locations $z_{m}$. \\
\hline$\varepsilon$ & Energy width of each energy channel. \\
\hline$w_{\mathrm{w}}, n_{\mathrm{w}}$ & $\begin{array}{l}\text { Energy width and number of channels of the sampled en } \\
\text { ergy window of the AES (Auger electron spectrometry) spec } \\
\text { trum. } n_{\mathrm{w}} \epsilon=w_{\mathrm{w}} \text {. }\end{array}$ \\
\hline$w^{\oplus}, n^{\oplus}$ & $\begin{array}{l}\text { Energy width and number of channels of the energy region } \\
\text { near the } J \text { maximum } J^{\oplus} \text { that are impacted by noise. } n^{\oplus} \varepsilon= \\
w^{\oplus} \text {. }\end{array}$ \\
\hline$w^{\ominus}, n^{\ominus}$ & Corresponding quantities for the minimum, $J^{\ominus}$. \\
\hline$w_{\sigma}, n_{\sigma}$ & $\begin{array}{l}\text { Energy width and number of channels of the base region } \\
\text { under the maximum } J^{\oplus} \cdot n_{\sigma} \varepsilon=w_{\sigma} \text {. }\end{array}$ \\
\hline$\tau_{\mathrm{I}}$ & Standard deviation of combined Gauss distributions. \\
\hline$R[v, \sigma]$ & $\begin{array}{l}\text { Average maximum of } v \text { numbers randomly drawn from a } \\
\text { centered Gauss distribution with standard deviation } \sigma \text {. }\end{array}$ \\
\hline$\Lambda[v]$ & $R[v, 1]$ \\
\hline$\left\langle S_{\infty}\right\rangle$ & Mean $S$ is region where $X=0$. \\
\hline$\sigma_{S_{\infty}}$ & Standard deviation of $S$ in zero-atom-fraction region. \\
\hline$z_{\infty}$ & Depth below which $X=0$, i. e. $z>z_{\infty} \Longrightarrow X=0$. \\
\hline
\end{tabular}

calibrated with a magnification standard.

The measurements were carried out with an electron probe diameter of $\approx 50 \mathrm{~nm}$ at the specimen surface. The measurement of carbon AFDPs is particularly challenging because of surface contamination by adventitious carbon, interfering with the measurement of the local carbon fraction in the specimen. To circumvent this problem, Auger electron spectra were recorded while constantly sputtering the specimen surface with Ar at a low flux rate, realized by a low pressure setting of the Ar sputter gun. While the resulting Ar flux density has not been quantified, the setting was empir-

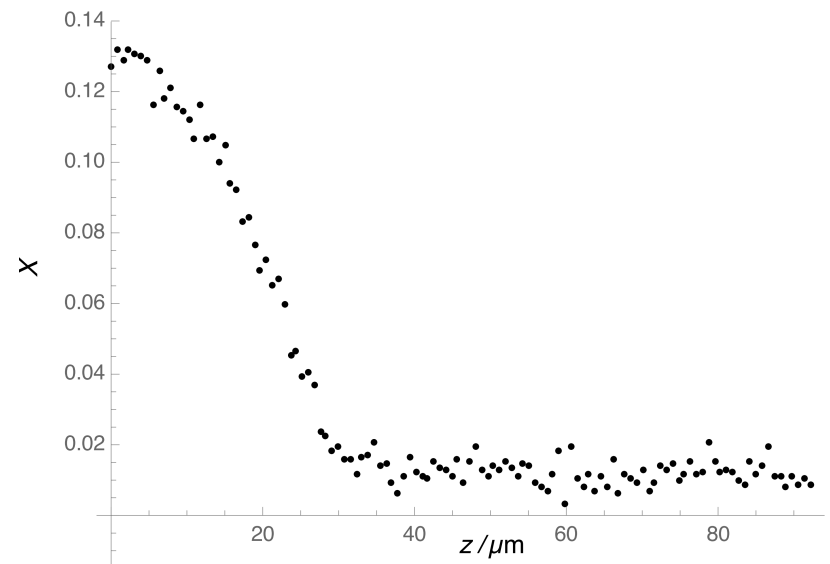

FIG. 2: Experimental AFDP (atom-fraction-depth profile) $X^{\mathrm{xp}}[z]$ of carbon in AISI-316L (austenitic stainless steel), SECIS (surface engineering by concentrated interstitial solute carbon, nitrogen) surface-engineered by low-temperature carburization from a gas phase. (Courtesy of Name removed for double-blind reviewing).

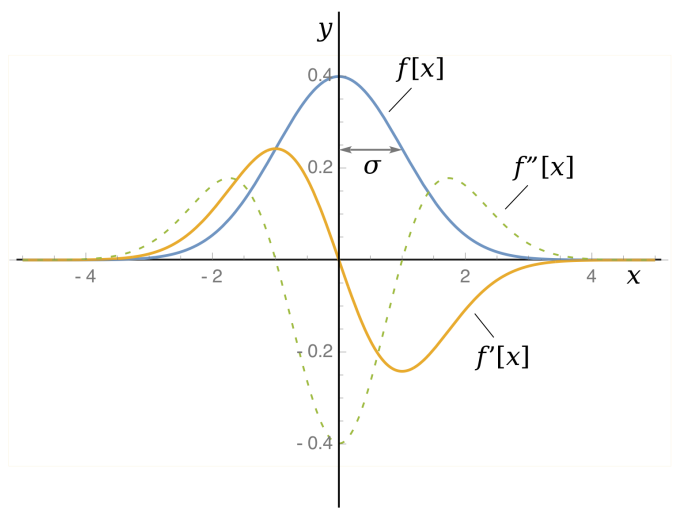

FIG. 3: Centered (mean value $\mu=0$ ) Gauss (or "normal") distribution with standard deviation $\sigma(\sigma=1$ in this plot.) $f[x]$ is the PDF (probability density function), $f^{\prime}[x]$ its first derivative, and $f^{\prime \prime}[x]$ its second derivative.

ically optimized to perform effective surface cleaning while minimizing sputtering of the actual specimen, which might generate artifacts of preferential sputtering. With the setting we have employed, the systematic error that may still arise from preferential sputtering and/or surface roughening after sputtering for a long time is sufficiently small, and the general efficacy of such "dynamic" sputtering has been independently demonstrated by our group (unpublished). The spectra obtained in this way were quantitatively evaluated in terms of local carbon fraction ${ }^{\mathrm{xp}} X[z]$ by comparison with calibration spectra we obtained from a suite of $\mathrm{Fe}-\mathrm{C}$ alloys with known compositions under equivalent 

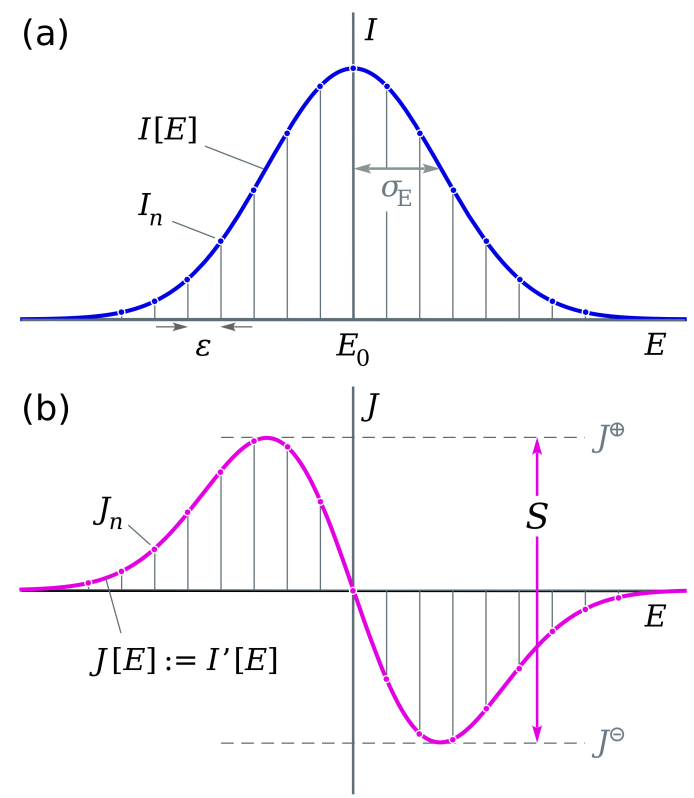

FIG. 4: Model of signal extraction from a discretized Auger electron spectrum $J_{n} \equiv J\left[E_{n}\right]$. (a) The peak is modeled as the PDF (probability density function) of a centered Gauss ("normal") distribution with mean value $E_{0}=0$ (without limiting generality) and standard deviation $\sigma_{\mathrm{E}}$. The large background that occurs in practice is neglected here. (b) First derivative $\left.J_{n} \equiv(\mathrm{d} J / \mathrm{d} E)\right|_{E=E_{n}}$. The quantity from which elemental concentrations are derived in SAM is $S:=\left(\operatorname{Max}\left[J_{n}\right]-\operatorname{Min}\left[J_{n}\right]\right)$.

experimental conditions.

Different from a smooth and monotoneously decreasing theoretical fraction-depth profile ${ }^{\text {th }} X[z]$ that would be expected as a result of diffusion, ${ }^{9}$ the experimentally determined ${ }^{\mathrm{xp}} X[z]$ in Fig 2 exhibits random local fluctuations. Even reducing the noise by locally averaging over these fluctuations (e.g. by least-square fitting a function ${ }^{\mathrm{nr}} X[z]$ to the data points, not shown in Fig 2) does not yield a profile compatible with the predictions of diffusion theory. As seen in Fig 2, the experimentally determined ${ }^{\mathrm{xp}} X[z]$ suffers from an additional systematic error, which is the focus of this study: a background ${ }^{{ }^{b g}} X[z]$ that causes ${ }^{\mathrm{nr}} X[z]$ to remain at a significant finite level $(\approx 0.01)$, even in the region of the alloy "core," i. e. at depths $z>z_{\infty}$ into which no such levels of carbon could have possibly diffused under the applied processing conditions. On the other hand, ${ }^{{ }^{b}} X[z]$ cannot be of the same magnitude in the regions of high $X$ near the surface $(z \gtrsim 0)$, as the level of ${ }^{n r} X[z]$ displayed here agrees well with the results of other, independent experimental meth-
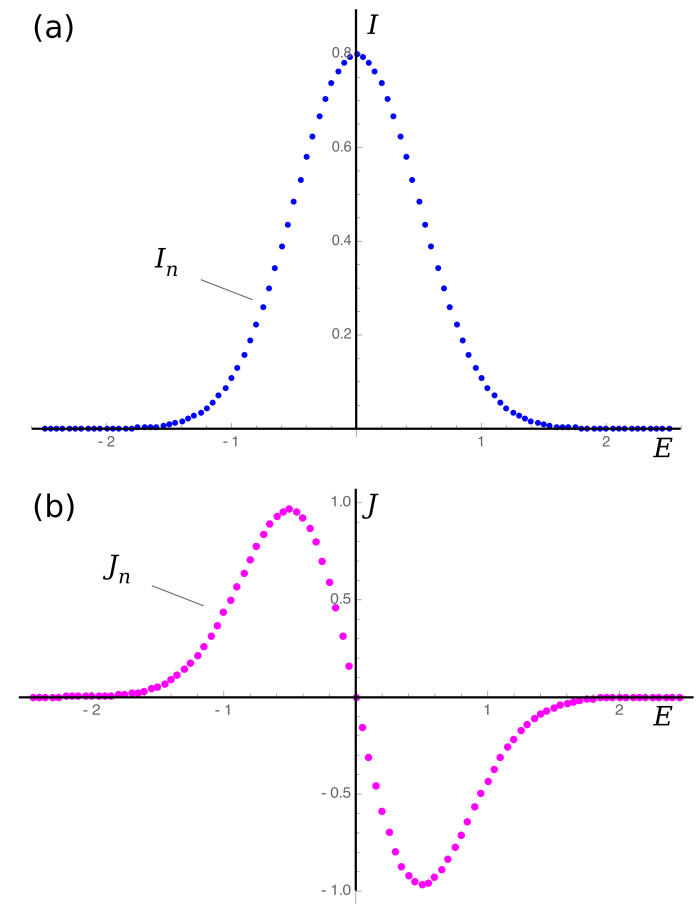

FIG. 5: Simulation of AES peak processing. (a) Peak in an AES spectrum modeled as discretized PDF (probability density function) of a Gauss distribution. (The numerical values and units of $E, I$ and derived quantities are of no interest.) (b) First derivative $J_{n}$, obtained by numerical differentiation according to (10).

ods by which we analyzed the near-surface level of carbon. It is also apparent from Fig. 2 that the random fluctuations in ${ }^{{ }^{x}} X[z]$ are stronger in regions of low $X$ than in regions of high $X$. As $X_{\mathrm{C}} \nless 0$, stronger fluctuations imply an increased (minimum) background level. In conclusion, the local background level (local average background) ${ }^{\text {bg }} X[z]$ ) depends on the local carbon fraction, ${ }^{{ }^{b g}} X={ }^{b g} X[X]$.

While noise in AES spectra has been discussed extensively in the literature, ${ }^{10}$ information on concentrationdependent background correction appears to be limited. The following section presents a theory that explains the observed background as a combined result of statistical noise and the differentiation by which AES spectra are processed to obtain the local atom fraction ${ }^{\mathrm{xp}} X$. The theory leads to a practical procedure for stripping the background ${ }^{{ }^{b}} X$ from SAM AFDPs. The validity of this approach is verified by applying it to simulated data. Subsequently, the approach is applied to the experimental data of Fig 2. 
(a)

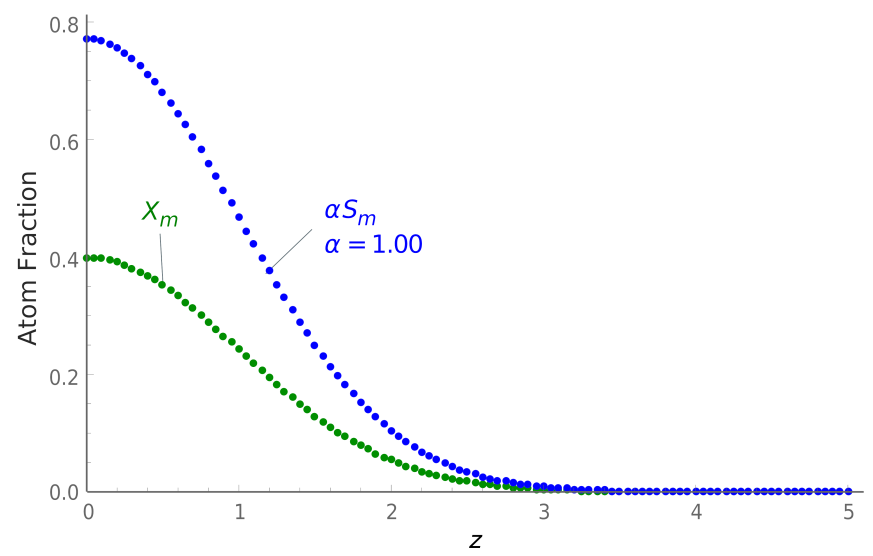

(b)

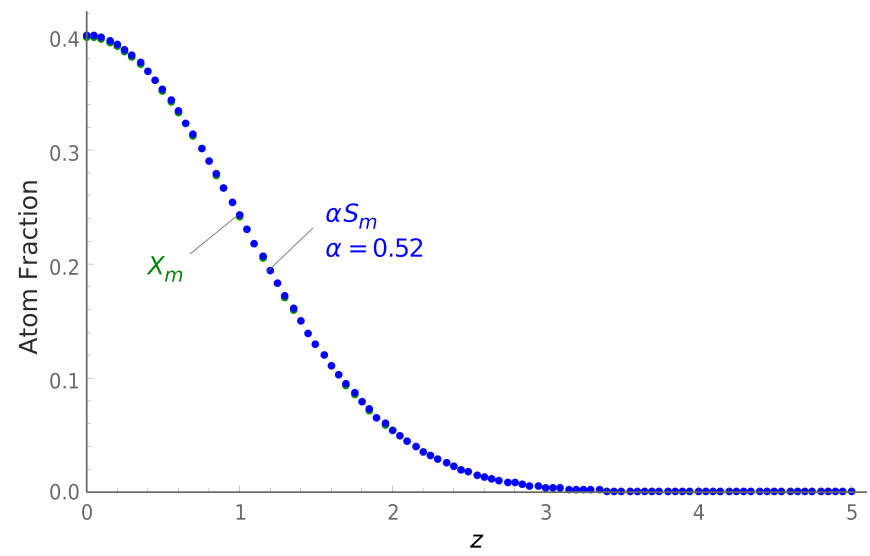

FIG. 6: Simulation of AES peak processing. (a) $X_{m}$ : Assumed hypothetical AFDP. $\alpha S_{m}$ : signal obtained from the derivative data in Fig 5b according to Fig $4 \mathrm{~b}$ with $\alpha=1.00$. (b) Same as (a), but with $\alpha=0.52$. The graphs perfectly coincide. (Note change of ordinate scale compared to (a).)

\section{MODEL}

In the discretized electron-energy spectra $I_{n} \equiv I\left[E_{n}\right]$ that are recorded from materials surfaces for the purpose of compositional analysis, the Auger electron peaks that serve for compositional analysis by SAM are usually very small features compared to the local background. To safely discriminate between peak and background, therefore, the spectra $I_{n}$ are differentiated with regard to the energy $E$ to obtain the derivative $J_{n}:=\left.(\mathrm{d} I / \mathrm{d} E)\right|_{E=E_{n}}$. The primary signal is the slope-extrema difference between the maximum and the minimum $J_{n}::^{10}$

$$
S:=J^{\oplus}-J^{\ominus},
$$

where

$$
\begin{aligned}
J^{\oplus} & :=\operatorname{Max}_{n}\left[J_{n}\right], \\
J^{\ominus} & :=\operatorname{Min}_{n}\left[J_{n}\right] .
\end{aligned}
$$

Generally, for a material containing $N$ different elements, the local atom fraction $X^{(i)}$ of element $i$ can be quantified from AES spectra by ${ }^{10,11}$

$$
X^{(i)}=\frac{S^{(i)} /{ }^{\circ} S^{(i)}}{\sum_{k=1}^{N}\left(S^{(k)} /{ }^{\circ} S^{(k)}\right)},
$$

where the $S^{(k)}$ are the local signals (1) measured for the different elements and the ${ }^{\circ} S^{(k)}$ are (absolute) sensitivity factors, representing the signals that corresponding pure-element standards produce under equivalent conditions. ${ }^{10}$

A variable local fraction $X \equiv X[z]$ of carbon in a stainless steel matrix with otherwise constant composition can be described as a binary system of carbon atoms and "metal" atoms, where the latter are a fixed combination of $M$ relevant atom species ( $\mathrm{Fe}, \mathrm{Cr}, \mathrm{Ni}, \ldots$ ). Denoting the metal atom fraction as $X^{\mathrm{M}} \equiv X^{\mathrm{M}}[z]$,

$$
X+X^{\mathrm{M}}=1 .
$$

Combining (4) with (5) yields

$$
\frac{X}{1-X}=\frac{S /{ }^{\circ} S}{\sum_{k=1}^{M}\left(S^{(k)} /{ }^{\circ} S^{(k)}\right)}:=\alpha S .
$$

Since the background correction that is the objective of this work is mainly needed in regions of small carbon fraction (Fig 2), the model can further be simplified by assuming $X \ll 1$, which linearizes (6) into

$$
X=\alpha S
$$

Carbon is interstitially dissolved in the austenite matrix provided by the metal atoms. If there were no expansion of the austenite lattice parameter with $X$ and if there were no instabilities causing variation of the electron dose underlying individual spectra, the signals $S^{(k)}$ of the metal atoms would be constant for every spectrum of the linescan. In reality, there are beam instabilities and there is a weak positive correlation of the lattice parameter with $X$. With the experimentally determined coefficient, ${ }^{8}$ the decrease of the $S^{(k)}$ will be $\lesssim 3 \%$ relative in regions where $X \lesssim 0.05$ that 
are most relevant for the background correction to be developed here. Both effects, the variation of the electron dose and lattice parameter expansion, can be corrected for - independent of $X$ - by adjusting $\alpha$ for each spectrum based on the measured $S^{(k)}$. However, to keep expressions simpler, the following assumes that $\alpha$ is constant.

For the purpose of modeling, neglect the background and assume that the original Auger electron peak $I[E]$ corresponds to the PDF (probability density function) of a Gauss ("normal") distribution (Fig 3) with standard deviation $\sigma_{\mathrm{E}}$, centered at some energy $E_{0}$, as shown in Fig $4 \mathrm{a}$. Since the peak location $E_{0}$ is of no further interest, let $E_{0}=0$. Then,

$$
I[E]=\beta X \frac{1}{\sigma_{\mathrm{E}} \sqrt{2 \pi}} \operatorname{Exp}\left[-\frac{1}{2}\left(\frac{E}{\sigma_{\mathrm{E}}}\right)^{2}\right],
$$

where the "peak strength factor" $\beta$ is a factor of the constant $\alpha$. The first derivative has the form

$$
J[E]=-\beta X \frac{E}{\sigma_{\mathrm{E}}^{3} \sqrt{2 \pi}} \operatorname{Exp}\left[-\frac{1}{2}\left(\frac{E}{\sigma_{\mathrm{E}}}\right)^{2}\right] .
$$

The corresponding graph is shown in Fig 4b, which also visualizes how the signal $S$ is obtained from $J[E]$.

In reality, $J[E]$ is acquired by an MCA (multichannel analyzer), yielding counts of Auger electrons within in energy intervals of constant width $\varepsilon$ (schematically indicated in Fig 4). The instrument we have used for the experimental work obtains the discretized derivative $J_{n}$ by a noise-reducing algorithm that evaluates 9 energy channels and then obtains $S$ from the difference between the maximum and the minimum of this noise-reduced derivative. For the current purpose of understanding the impact of noise in $I$ on $X_{m}=\alpha S_{m}$, it is sufficient to obtain $J_{n}$ as "standard" numerical derivative

$$
J_{n}=\frac{I_{n+1}-I_{n-1}}{2 \varepsilon} .
$$

For modeling noise, random fluctuations $\Delta I_{n}$ are added to $I_{n}$ in each channel $n$ equally and independently, described by a continuous random variable $\Delta I$ with the underlying PDF

$$
p_{\Delta I}[x]=\frac{1}{\sigma_{\mathrm{I}} \sqrt{2 \pi}} \operatorname{Exp}\left[-\frac{1}{2}\left(\frac{x}{\sigma_{\mathrm{I}}}\right)^{2}\right],
$$

i. e. a Gauss distribution with standard deviation $\sigma_{\mathrm{I}}$.
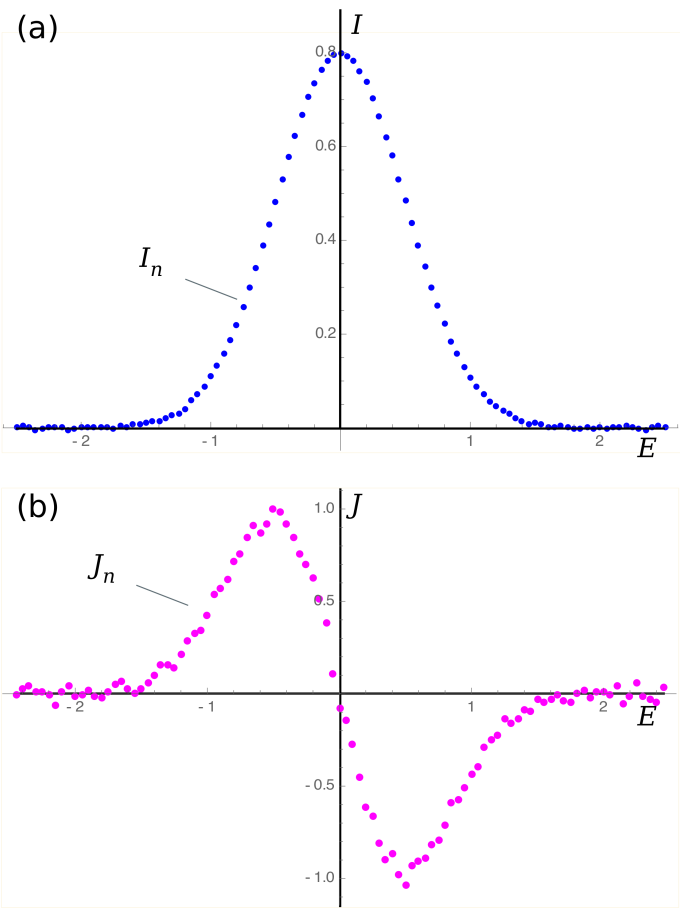

FIG. 7: Simulation of AES peak processing. (a) Like Fig 5a, but with noise added $\left(\sigma_{I}=0.002\right)$. The noise is barely visible. (b) First derivative $J_{n}$ (corresponding to Fig 5b), clearly showing the presence of noise.

\section{SIMULATION}

\section{A. Noise Analysis}

A Mathematica12 "manipulator," coded in Wolfram language, served to simulate and study the impact of random fluctuations on $S$ obtained by numerical differentiation. Here and in the following, the units of $E$ and $I$ and the quantities derived from them play no further role and are therefore ignored.

Figure 5 shows a discretized Gauss function that was set up as a model for a discretized intensity profile $I_{n}$ still without noise - and the corresponding numerical derivative $J_{n}$ as obtained by (10).

The graph labeled $X_{m}$ in Fig 6a is a hypothetical discretized carbon fraction profile $X_{m} \equiv X\left[z_{m}\right]$, i. e. the carbon fractions $X$ at equi-spaced locations $z_{m}$. For simplicity, this profile was also assumed to be (the positive branch of) a Gauss function, rather than a correct solution to the diffusion equation. The othe graph is a corresponding plot of $\alpha S_{m}$, obtained by pointwise (i) evaluation of the peak shape (8) with $\beta=1$, (ii) calculating the numerical derivative (10), (iii) determining $S_{m}$ according to (1), and (iv) multiplying $S_{m}$ 


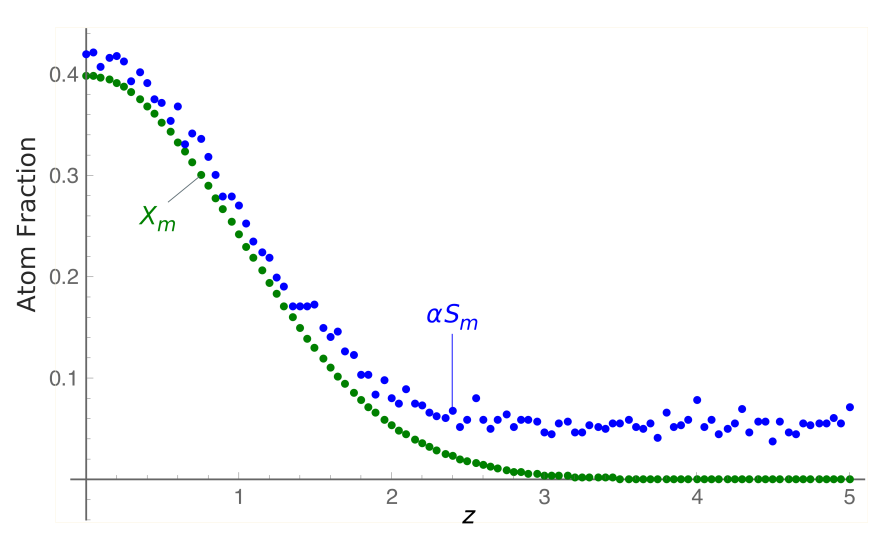

FIG. 8: Simulation of AES peak processing. $X_{m}$ : Assumed hypothetical fraction-depth profile. $\alpha S_{m}$ : Signal obtained from the derivative data in Fig $7 \mathrm{~b}$ with $\alpha=0.52$ and $\sigma_{J}=0.002$.

with $\alpha=1.00$. This reproduces the characteristics of the hypothetical profile, but with compressed shape. However, as shown in Fig 6b, $\alpha S_{m}$ exactly reproduces the hypothetical $X_{m}$ when choosing $\alpha=0.52$. This result confirms that the difference between maximum and minimum peak slope is a valid measure for the integrated intensity of a peak with constant shape, which, of course, is a consequence of the linearity of differentiation: Multiplying a peak $I[E]$ with a factor $\gamma$ will correspondingly increase its slope $J[E]$ at every $E$ and thus increase $S[E]$ by the same factor $\gamma$.

With the simulation set up as in Figs 5 and 6, it can now be investigated how adding noise to the discretized peak $I_{n}$ of Fig 5 a impacts the profile $\alpha S_{m}$ in Fig 6. Figures 7 and 8 show the same graphs as Figs 5 and 6 , but with random noise values $\Delta I_{n}$ added to each energy channel, drawn from the distribution (11) with $\sigma_{\mathrm{I}}=0.0015$. (While $\sigma_{\mathrm{I}}$ has the dimension of "electron counts" or "intensity," the absolute magnitude and unit are not important here.) The noise is barely visible in the graph of $I_{n}$, but clearly visible in the graph of $J_{n}$.

The corresponding $\alpha S_{m}$, obtained from $I_{n}+\Delta I_{n}$, i. e. in the presence of peak noise, is shown in Fig 8. This graph reproduces important features of the experimental data in Fig 2:

1. Over the entire range of $z_{m}, \alpha S_{m} \geq X_{m}$.

2. The lowest background is observed at $z=0$, where $X$ has its maximum.

3. With decreasing $X$ (increasing $z$ ), the background introduced by random noise $\Delta I$ increases.
4. The highest background is observed the right end ("tail") of the profile. Here, where $X_{n}=0, \alpha S_{m}$ features a constant, non-vanishing mean level $\alpha\langle S\rangle_{m}>0$.

How can these features be explained?

1. Regardless of the local $X_{m}$, the peak in $I_{n}$ at every location $z_{m}$ is sampled over $n_{\mathrm{w}}$ energy channels of constant width $\varepsilon$, i. e. an energy window of width $w_{\mathrm{w}}=n_{\mathrm{w}} \varepsilon$.

2. Adding random noise $\Delta I_{n}$ to $I_{n}$ will add random variations $\Delta J_{n}$ to $J_{n}$.

3. While noise generally changes $I_{n}$ and its slope $J_{n}$ everywhere within this window, the slopeextrema difference $S$ (from which $X$ is to be inferred) will typically be impacted only by $J_{n}$ variations in certain energy channels. These are the $n^{\oplus}+n^{\ominus}$ channels so close to the locations $E^{\oplus}, E^{\ominus}$ of the original extrema $J^{\oplus}, J^{\ominus}$ that their values may become the new extrema $J^{\oplus}, J^{\ominus}$ after adding $\Delta J_{n}$. In channels farther away from the original $E^{\oplus}, E^{\ominus}$, conversely, adding $\Delta J_{n}$ will typically not make their values comparable to $J^{\oplus}, J^{\ominus}$, so the added noise in those channels cannot change $S$.

4. In $z$ regions of high $X$, the peak in $I_{n}$ is high and $J_{n}$ has pronounced extrema $J^{\oplus}, J^{\ominus}$. The number $n^{\oplus}+n^{\ominus}$ of channels with values less than the typical $\Delta J$ away from $J^{\oplus}, J^{\ominus}$ is small (compared to the total number $n_{\mathrm{w}}$ of channels in the sampled window). Correspondingly, only $n^{\oplus}+n^{\ominus}$ of the randomly drawn $\Delta I_{n}$ and corresponding $\Delta J_{n}$ contribute to $\Delta S$. The probability that one of these randomly drawn $\Delta I_{n}$ and the resulting $\Delta J_{n}$ are far from their mean value of 0 implied by (11) is correspondingly limited. This is why Fig 8 features only a moderate average background level in regions of high $X$. Obviously, even in the regions of highest $X, n^{\oplus}+n^{\ominus}$ is large enough to ensure that $\Delta S>0$.

5. In $z$ regions of lower $X$, the peak in $I_{n}$ is low. $J_{n}$ has less pronounced extrema $J^{\oplus}, J^{\ominus}$ and is flatter in the regions around the locations $E^{\oplus}, E^{\ominus}$ of the extrema. Therefore, more energy channels have values less than the typical $\Delta J$ away from $J^{\oplus}, J^{\ominus}$, meaning that the fluctuations $\Delta J$ of a larger number $n^{\oplus}+n^{\ominus}$ of channels can contribute to $\Delta S$. 
Increased $n^{\oplus}+n^{\ominus}$ means increasing the number of random drawings that can contribute to $\Delta S$. A large number of drawings will likely produce a larger maximum $J^{\oplus}$ and a lower minimum $J^{\ominus}$ than a small number of drawings, thus a larger increase $\Delta S$ of $S$. This is why the average background increases with decreasing $X$ in Fig 8 .

6. The strongest noise-induced increase of $S$ is expected where $X[z]=0$, at the right end of the profile in Fig 8. Here, each one of the $n_{\mathrm{w}}$ energy channels can become the new maximum $J^{\oplus}$ or minimum $J^{\ominus}$ of $J_{n}$ and therefore impact $\Delta S$. Since any given $\Delta J_{n}$ can only increase $J^{\oplus}$ and decrease $J^{\ominus}$, noise will increase every $S_{m}$ : $X_{m}=0 \Longrightarrow \Delta S_{m}>0$. Since $\Delta S$ now follows from the maximum possible number $n_{\mathrm{w}}$ of random drawings, the average background in this region will be the highest - as seen in Fig 8 .

7. In regions of $X=0$, the background level is determined by the total number $n_{\mathrm{w}}$ of energy channels. In regions of significant non-vanishing levels of $X$, in contrast, the background level is determined by the peak shape, regardless of $n_{\mathrm{w}}$. At the border between these two regimes, the background level may feature a corresponding discontinuity.

\section{B. Quantitative Theory}

Based on these insights, the following section aims to develop a quantitative theory for the average background level. According to (10), noise $\Delta I$ in $I[E]$ alters the peak slope in each energy channel $E_{n}$ by

$$
\Delta J_{n}=\frac{\Delta I_{n+1}-\Delta I_{n-1}}{2 \varepsilon},
$$

where $\Delta I_{n+1}$ and $\Delta I_{n-1}$ are - by assumption - independent values of the random variable $\Delta I$ with the Gauss PDF (11). As shown in probability theory, the difference (or sum) of two random variables that are Gauss-distributed with standard deviations $\sigma_{1}, \sigma_{2}$, respectively, is a random variable following a Gauss distribution with standard deviation $\sqrt{\sigma_{1}^{2}+\sigma_{2}^{2}}$. Therefore, $\Delta J$ is a continuous random variable with the underlying PDF

$$
p_{\Delta J}[x]=\frac{1}{2 \varepsilon} \frac{1}{\tau_{\mathrm{I}} \sqrt{2 \pi}} \operatorname{Exp}\left[-\frac{1}{2}\left(\frac{x}{\tau_{\mathrm{I}}}\right)^{2}\right],
$$

where $\tau_{\mathrm{I}}:=\sqrt{2} \cdot \sigma_{\mathrm{I}}$.
First, consider regions where $X=0 \Longrightarrow J_{n}=0$. For AFDPs of the type shown in Fig 2, this will be true for $z>z_{\infty}$, where $z_{\infty}$ marks the beginning of the non-carburized alloy core. As stated earlier, noise in every energy channel $E_{n}$ included in the energy window $w_{\mathrm{w}}=\neq \varepsilon$ can contribute to $\Delta S$ in the same way. The most likely - average - noise-induced change $\Delta J^{\oplus}$ of the slope maximum will correspond to the most likely maximum $R$ of $n_{\mathrm{w}}$ numbers randomly drawn from the Gauss distribution (13). Since $J_{n}=0$ before noise, the new maximum is identical to the noise-induced change:

$$
\begin{aligned}
\left\langle J^{\oplus}\right\rangle & =\left\langle\Delta J^{\oplus}\right\rangle \\
& =: \frac{1}{2 \varepsilon} R\left[n_{\mathrm{w}}, \tau_{\mathrm{I}}\right],
\end{aligned}
$$

where " $\langle\ldots\rangle$ " denotes the mean value. Owing to the symmetry of (13), the most likely noise-induced change of $J^{\ominus}$ will be

$$
\begin{aligned}
\left\langle J^{\ominus}\right\rangle & =\left\langle\Delta J^{\ominus}\right\rangle \\
& =-\frac{1}{2 \varepsilon} R\left[n_{\mathrm{w}}, \tau_{\mathrm{I}}\right] .
\end{aligned}
$$

This implies

$$
\Delta S=\frac{1}{\varepsilon} R\left[n_{\mathrm{W}}, \tau_{\mathrm{I}}\right]
$$

\section{Lambda Function}

Figure 9 presents numerical data (obtained by Mathematica) for the average maximum $R$ of $v$ numbers drawn from a centered Gauss distribution with standard deviation $\sigma=1$, obtained by averaging over 1000 drawings for each $v$.

Another way to obtain this graph is the following: To be determined is the mean value $R$ of the maximum of $v$ numbers randomly drawn from a centered Gauss distribution with standard deviation $\sigma$. The condition that the maximum of $v$ randomly numbers equals $r$ corresponds to the event that $x_{n} \leq r$ for $i=1 \ldots v$. The probability of drawing one of the $x_{n} \leq r$ corresponds to $\Phi[r]$, that is the CDF (cumulative density function) of the distribution at $r$. The probability to draw $v$ numbers $x_{v}$ less that $r$, i. e. $x_{v} \leq r$ for $i=1 \ldots v$, is $\Phi[r]^{v}$. The mean maximum $R$ is the $r$ for which

$$
(\Phi[r])^{v}=\frac{1}{2} .
$$

For the $r$ that solves this equation, the probability to find all $x_{v} \leq r$ in a sample of $v$ randomly drawn 


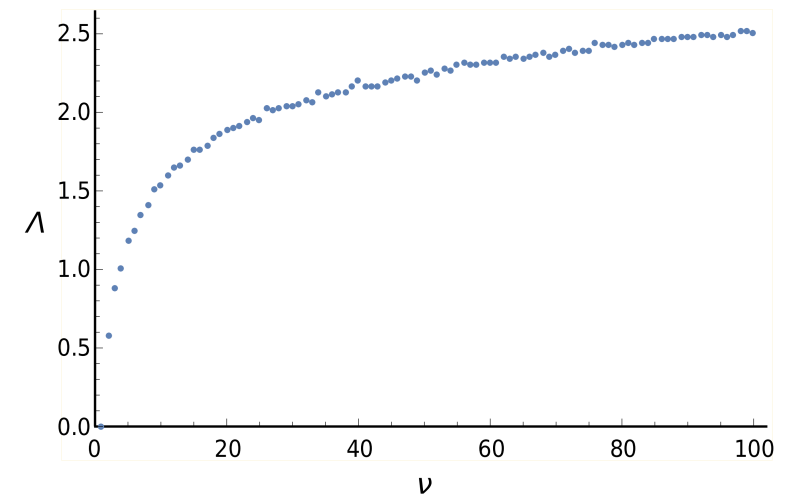

FIG. 9: Plot of the function $\Lambda[v]$, defined as average maximum of $v$ numbers drawn from a centered Gaussdistribution with standard deviation $\sigma=1$. These data were obtained numerically by averaging over 1000 drawings for each $v$.

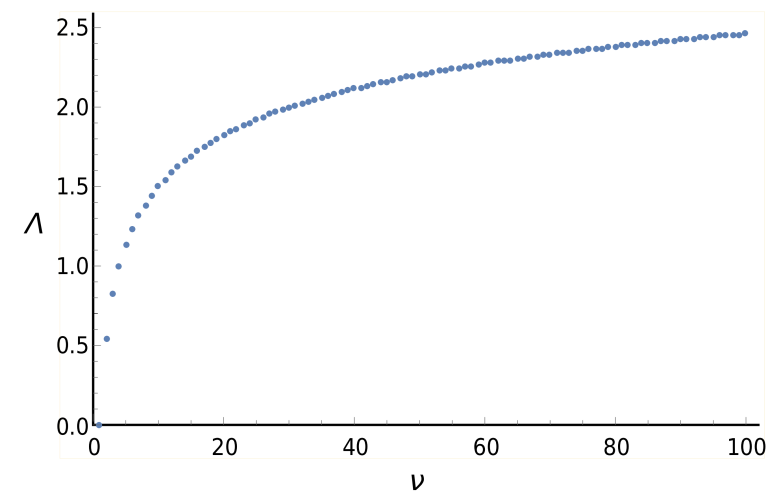

FIG. 10: Plot of the function $\Lambda[v]$, defined as average maximum of $v$ numbers drawn from a centered Gaussdistribution with standard deviation $\sigma=1$. These data were obtained by numerically solving (19) with (20).

numbers is $1 / 2$, i. e. it is equally likely for this to occur as it is for this not to occur. The CDF of a centered $(\mu=0)$ Gauss distribution is

$$
\Phi[x]:=\frac{1}{\sqrt{2 \pi}} \int_{-\infty}^{x} \operatorname{Exp}\left[-\frac{t^{2}}{2}\right] \mathrm{d} t
$$

While (19) cannot be solved for $r$ analytically, highly accurate solutions can be effectively obtained by numerical methods of equation solving. Figure 10 shows a plot of $R[v]$ calculated with this method. The graph agrees very well with the one in 9 (but is smoother).

Let $R[v, \sigma]$ denote the most likely maximum of $n$ drawings from a centered Gauss distribution with standard deviation $\sigma$. Since the width of the Gauss distri-

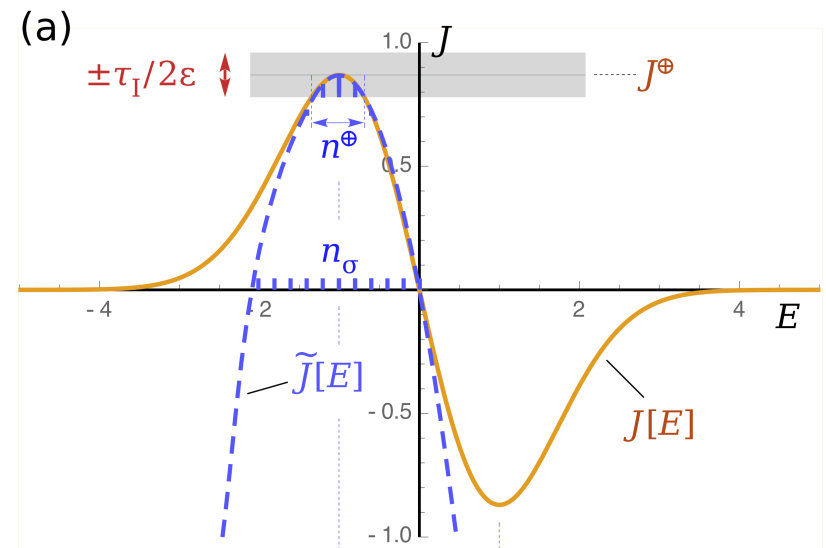

(b)
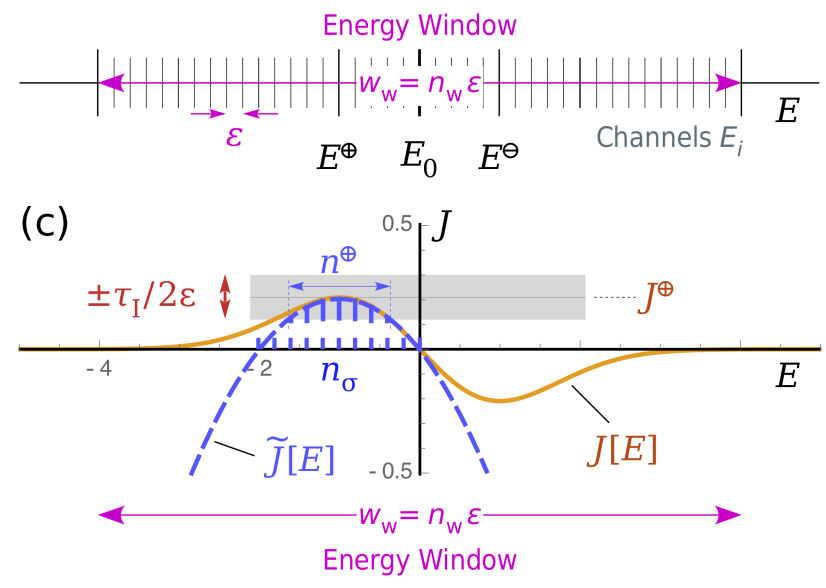

FIG. 11: Number $n^{\oplus}+n^{\ominus}$ of energy channels in the peak derivative $J[E]$ in which noise can alter the maximum of $J[E]$. Only the $n^{\oplus}$ channels near the maximum $J^{\oplus}$ at $E^{\oplus}$ are illustrated. The $n^{\ominus}$ channels near the minimum $J^{\ominus}$ at $E^{\ominus}$ are not shown. (a) Strong peak: the maximum can be altered by noise in only few channels. (b) Legend indicating energy channels and the energy window width $w_{\mathrm{w}}$. (c) Weak peak: the maximum can be altered by noise in many channels.

bution scales linearly with $\sigma$,

$$
2 R\left[v, \tau_{\mathrm{I}}\right]=: 2 \tau_{\mathrm{I}} \Lambda[v],
$$

where $\Lambda[v]$ is defined as $R[v, 1]$, as plotted in Figs 9 and 10 .

\section{Energy Channels of Potential Impact}

Figure 11 illustrates how to determine the likely number $n_{\oplus}+n_{\ominus}$ of energy channels around $E^{\oplus}$ and $E^{\ominus}$ in which noise can impact the slope-extrema difference $S$. These are the $E_{n}$ for which $J_{n}$ is closer than $\tau_{\mathrm{I}}$ (the typical noise fluctuation of $J_{n}$ ) to the maximum $J^{\oplus}$ at $E^{\oplus}$ or the minimum $J^{\ominus}$ at $E_{\ominus}$, respectively. Figure 11 
only shows the analysis for $n_{\oplus}$. Owing to the inversion symmetry of $J[E], n_{\ominus}=n_{\oplus}$.

To obtain an analytic expression for $n_{\oplus}$ as a function of $\tau_{\mathrm{j}}, J[E]$ can be approximated by a negative parabolic function

$$
\widetilde{J}[E]:=J^{\oplus}-\frac{J^{\oplus}}{\sigma_{\mathrm{E}}^{2}}\left(E+\sigma_{\mathrm{E}}\right)^{2} .
$$

Figure 11 shows the graph of $\widetilde{J}[E]$ as dashed lines. The coefficients in (22) are chosen such that

$$
\begin{aligned}
\widetilde{J}_{\oplus}:=\tilde{J}\left[E^{\oplus}\right] & =J^{\oplus}, \\
\widetilde{J}[0] & =0 .
\end{aligned}
$$

As can be seen in the figure, $\widetilde{J}[E]$ fits $J[E]$ very well particularly near the maximum $J\left[E^{\oplus}\right]$, which is important for estimating $n_{\oplus}$. From the graph of $\widetilde{J}[E]$, it is apparent that $\tilde{J}^{-1}[E]$, the inverse function of $\widetilde{J}[E]$, is a square-root-like function for $J \leq J^{\oplus} . n^{\oplus}$ is the solution to the equation

$$
\widetilde{J}\left[E^{\oplus}+\frac{1}{2} n_{\oplus} \varepsilon\right]=J^{\oplus}-\frac{\tau_{\mathrm{I}}}{2 \varepsilon} .
$$

The solution is

$$
n^{\oplus}=n_{\sigma} \sqrt{\frac{\tau_{\mathrm{I}} / 2 \varepsilon}{J^{\oplus}}} .
$$

For $n^{\oplus}$, the inversion symmetry of $J[E]=-J[-E]$ implies

$$
n^{\ominus}=n^{\oplus} .
$$

As long as $J[E] \gg \Lambda N \tau_{\mathrm{I}} / 2 \varepsilon$, therefore,

$$
\begin{gathered}
\Delta S=\Lambda\left[n^{\oplus}\right] \frac{T_{\mathrm{I}}}{2 \varepsilon}+\Lambda\left[n^{\ominus}\right] \frac{T_{\mathrm{I}}}{2 \varepsilon} \\
\stackrel{(27)}{=} 2 \Lambda\left[n^{\oplus}\right] \frac{T_{\mathrm{I}}}{2 \varepsilon} .
\end{gathered}
$$

Fig 11 illustrates how the number of channels that can impact the maximum (or minimum) increases as the slope-extrema difference $S$ decreases. According to (26), $E^{\oplus} \rightarrow \tau_{\mathrm{I}} / 2 \varepsilon$ should result in $n^{\oplus} \rightarrow n_{\sigma}$. However, this regime will not be reached since the background $\Lambda[N] \tau_{\mathrm{I}} / 2 \varepsilon$ caused by all channels is higher than $\tau_{\mathrm{I}} / 2 \varepsilon$. Thus, as $J^{\oplus} \rightarrow \Lambda[N] \tau_{\mathrm{I}} / 2 \varepsilon, 2 n_{\sigma} \rightarrow n_{\mathrm{w}}$. Under this condition, noise from any of the $n_{\mathrm{w}}$ energy channels within the window $w_{\mathrm{w}}=n_{\mathrm{w}} \varepsilon$, including those around the location $E^{\ominus}$ of $J^{\ominus}$, begins to contribute to changes of the maximum within the window (and conversely for the minimum). So, for $\Lambda[N] \tau_{\mathrm{I}} / 2 \varepsilon>\left(J^{\oplus},-J^{\ominus}\right)>0$, the maximum and minimum at $E^{\oplus}, E^{\ominus}$ lose their meaning and $2 n_{\sigma} \rightarrow n_{\mathrm{w}}$.

\section{BACKGROUND REDUCTION PROCEDURE}

1. Determine the number $n_{\mathrm{w}}$ of energy channels within the sampled energy window $w_{\mathrm{w}}$ and the energy channel width $\varepsilon=w_{\mathrm{w}} / n_{\mathrm{w}}$.

2. Record a sufficiently long profile section from the core region, where $X=0$.

3. Determine the constant average background level $\alpha\left\langle S_{\infty}\right\rangle$ at the right end of the profile, where $X=0$.

4. Determine $\alpha \tau_{\mathrm{I}}$ from

$$
\begin{aligned}
\left\langle X_{\infty}\right\rangle & =\alpha\left\langle S_{\infty}\right\rangle \\
& =\alpha\left\langle\Delta S_{\infty}\right\rangle \\
& =\alpha \frac{\tau_{\mathrm{I}}}{\varepsilon} \Lambda\left[n_{\mathrm{w}}\right] .
\end{aligned}
$$

5. Determine

$$
\sigma_{\mathrm{E}}=\frac{E^{\ominus}-E^{\oplus}}{2}
$$

from the spacing between the locations $E^{\ominus}$ and $E^{\oplus}$ of the minimum and maximum of a differentiated peak.

6. Determine

$$
n_{\sigma}=\frac{\sigma_{\mathrm{E}}}{\varepsilon}
$$

7. For each $X_{m}$ of the experimental data, determine

$$
\begin{aligned}
n_{m}^{\oplus} & =n_{\sigma} \sqrt{\frac{\tau_{I} / 2 \varepsilon}{J_{m}^{\oplus}}} \\
& =n_{\sigma} \sqrt{\frac{\alpha T_{I} / 2 \varepsilon}{\alpha S_{m} / 2}} \\
& =n_{\sigma} \sqrt{\frac{\alpha T_{I} / \varepsilon}{X_{m}}} \\
& =n_{\sigma} \sqrt{\frac{2\left\langle X_{\infty}\right\rangle}{\Lambda[N] X_{m}}} .
\end{aligned}
$$

8. From each $X_{m}, z_{m}<z_{\infty}$, subtract

$$
\Delta X_{m}=\alpha \Delta S_{m}=2 \Lambda\left[n_{m}^{\oplus}\right] \alpha \frac{\tau_{\mathrm{I}}}{2 \varepsilon} .
$$

9. At the right end of the profile, where $z>z_{\infty}, X=$ 0 , subtract the average background level

$$
\alpha\left\langle S_{\infty}\right\rangle=\alpha \frac{\tau_{\mathrm{I}}}{\varepsilon} \Lambda\left[N_{\mathrm{E}}\right] .
$$




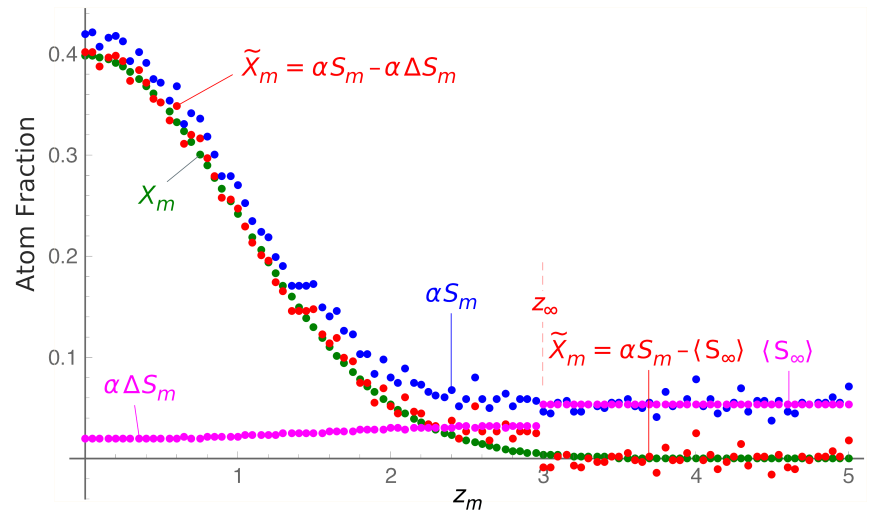

FIG. 12: Background subtraction. $X_{m}$ : hypothetical atomfraction depth profile of Fig 6. $\alpha S_{m}$ : noise-impacted profile of Fig 6. $\alpha \Delta S_{m}$ and $\left\langle S_{\infty}\right\rangle$ : Background according to (39) for $z<3$ and $\left\langle X_{\infty}\right\rangle$ for $z \geq 3$ according to (40). $\tilde{X}_{m}$ : $X$ after subtracting the background $\alpha \Delta S_{m}$ and $\left\langle S_{\infty}\right\rangle$.

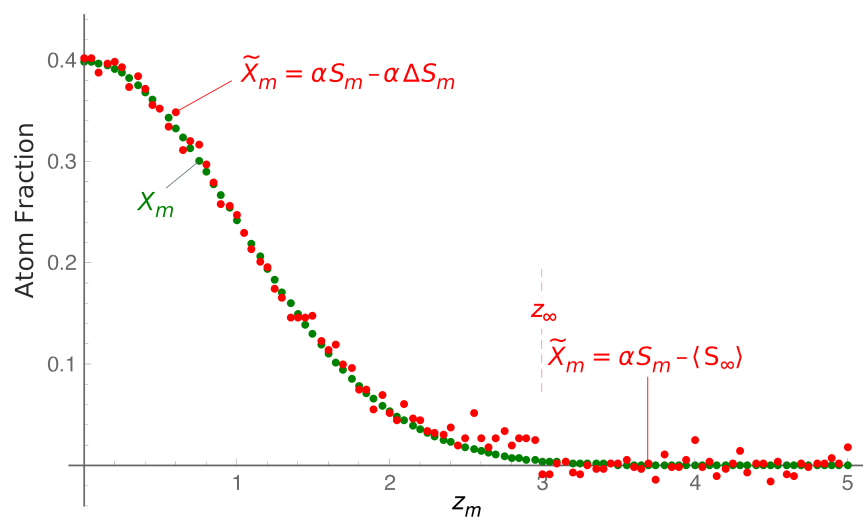

FIG. 13: Comparison between background-subtracted data $\left(\tilde{X}_{m}\right)$ of Fig 12 and original hypothetical fraction-depth profile $X_{m}$ of Fig 6 .

\section{VALIDATION}

To validate the background-reduction procedure of Section IV, consider the hypothetical $X_{m}$ and the noiseimpacted $\alpha S_{m}$ of Fig 8. Figure 12 reproduces $X_{m}$ and $\alpha S_{m}$ from Fig 6. For $z>z_{\infty}=3, \alpha S_{m}$ features a constant mean background level of $\left\langle X_{\infty}\right\rangle=0.06$. The graph $\tilde{X}_{m}$ was obtained by background subtraction according to (39) for $z<z_{\infty}$ and subtracting $\left\langle X_{\infty}\right\rangle$ for $z \geq z_{\infty}$ according to (40). Thus, the graph corresponds to

$$
\tilde{X}_{m}=\left\{\begin{array}{l}
\alpha S_{m}-\alpha \Delta S_{m} \text { for } z<z_{\infty} \\
\alpha S_{m}-\left\langle X_{\infty}\right\rangle \text { for } z \geq z_{\infty}
\end{array}\right.
$$

For better visibility, Fig 13 only shows the original hypothetical profile $X_{m}$ and the background-subtracted

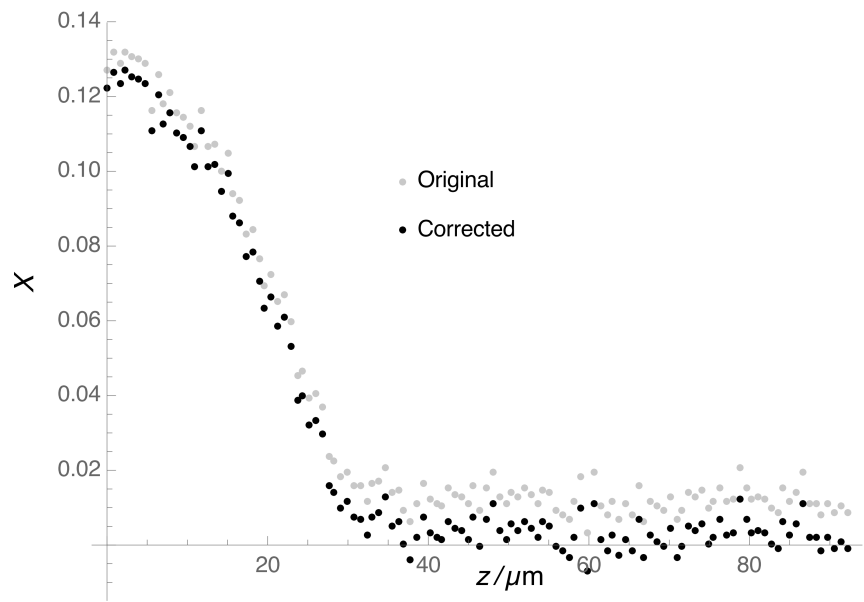

FIG. 14: Background subtraction for the experimental data of Fig 2.

profile $\tilde{X}_{m}$. Naturally, the background production did not eliminate the noise in $\alpha S_{m}$. One consequence of this fact is that in the region where $X_{m} \rightarrow 0, \tilde{X}$ features partly negative values. However, Fig 13 demonstrates that the original $X_{m}$ (green) constitutes a well-matching (e. g. minimizing least-square deviation), smooth fitting line for $\tilde{X}_{m}$. Accordingly, apart from the noise, the background reduction procedure has very well recovered the original hypothetical profile $X_{m}$.

\section{APPLICATION EXAMPLE}

As an application example, Fig 14 shows the result of applying the background subtraction algorithm of Section IV to the experimental SAM AFDP of Fig 2. The original data points are shown in light gray. This profile was recorded with the following parameters: $\varepsilon=1 \mathrm{eV}, n_{\mathrm{w}}=30, n_{\sigma}=11$. In the profile "tail" (i. e. the non-carburized alloy core), for $z>z_{\infty}=50 \mathrm{~nm}$, the mean measured carbon fraction is $\alpha\left\langle S_{\infty}\right\rangle=0.0117$ (Fig 16).

The data obtained after background subtraction are shown in black. Similar to Fig13, the backgroundsubtracted data in the core ("tail") region are obtained by subtracting $\alpha\left\langle S_{\infty}\right\rangle$ from the experimental data and therefore include negative values for $X$, such that the average in this region now corresponds to zero. Also similar to Fig 13, a lesser amount of background was subtracted in regions of higher $X$. In the region of maximum $X$ near $z=0$, the subtracted amount is only 0.005 , i. e. about $1 / 3$ of the correction in the tail region. 


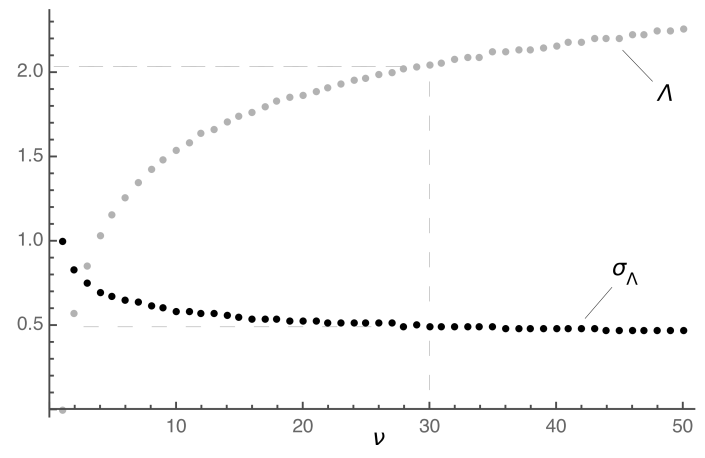

FIG. 15: $\Lambda[v]$ (compare Fig 9), i.e. mean maximum $\langle\operatorname{Max}[f[x]]\rangle$ of $v$ numbers drawn from a centered Gauss distribution $f[x]$ with standard deviation $\sigma=1$ and corresponding standard deviation $\sigma_{\Lambda}[v]$ characterizing the stochastic variation of $\langle\operatorname{Max}[f[x]]\rangle$ for the range $1 \leq v \leq$ 50. At $v=30, \sigma_{\Lambda} /\langle\operatorname{Max}[f[x]]\rangle=0.25$. Compare with Fig 16.

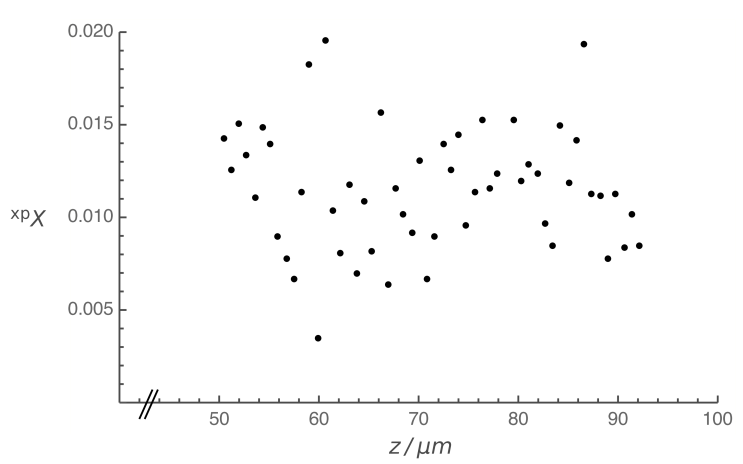

FIG. 16: "Tail" of the fraction-depth profile of Fig 2: The range of the graph where $z>50 \mathrm{~nm}$. It is known that this region is free of dissolved carbon. Mean value: $\alpha\left\langle S_{\infty}\right\rangle=$ 0.0117. Standard deviation: $\sigma_{S_{\infty}}=0.0035 . \sigma_{S_{\infty}} /\left(\alpha\left\langle S_{\infty}\right\rangle\right)=$ 0.30 .

\section{DISCUSSION}

Comparing the background subtraction performed in the tail of the AFDP of Fig 14 with the background subtraction performed in the region of maximum $X$ near $z=0$ illustrates the order of error that would be introduced by simply subtracting $\alpha\left\langle S_{\infty}\right\rangle$ everywhere: The atom fraction at the beginning of the profile would be underestimated by about 0.01 ( $1 \mathrm{at} \%)$.

One way to test that the elevated background level at low carbon fractions actually originates from the "channel statistics" phenomenon discussed here is to compare the observed scatter of the values in the tail region of the experimental profile with the theoretically ex-

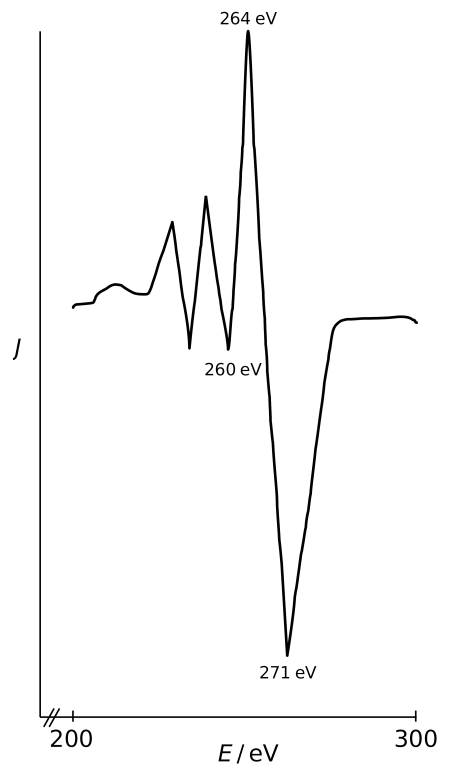

FIG. 17: AES signature of carbon in carbidic environment (TaC, after Handbook of $\mathrm{AES}^{13}$ ).

pected value. Figure 15 shows the theoretical standard deviation $\sigma_{\mathrm{b}}$ of the background signal expected for $v=$ 30 energy channels in the sampled window. The graph also includes the the function $\Lambda[v]$ (light gray dots), as shown in Fig 9. For $v=30$, as in the experimental example, the graphs predict $\Lambda[v] \approx 2.0$ and $\sigma_{\Lambda} \approx 0.5$. The ratio $\sigma_{\Lambda} / \Lambda[v] \approx 0.25$ matches very well with the corresponding ratio $\sigma_{\mathrm{b}} /\left(\alpha\left\langle S_{\infty}\right\rangle=0.0035 / 0.0117=0.30\right.$ for the scatter observed in Fig 16.

Figure 13 demonstrates that the approximations used in deriving the background correction procedure are well suited for an effective background correction within the framework of the model. In practice, this model will work well if the signal is dominated by one peak with the approximate shape of a Gauss-function. In reality, this may not always be the case. For carbon in carbidic environment, specifically, the differentiated Auger electron spectrum features one minimum and three distinct maxima in the energy range $[200 \mathrm{eV}, 300 \mathrm{eV}],{ }^{13}$ as illustrated in Fig 17. For reasons not well understood, there are experimental conditions under which the highest and second-highest maximum (or even all three maxima) become comparably high, thus impact $J^{\oplus}$ and, thus, $S$. According to the theory developed here, this will increase the background as noise in the channels near 3 (or 4) extrema contributes to the background - compared to just 2 extrema assumed in the model. In principle, this can be easily 
accomodated in the model, however not if the relative height of the maxima or the underline peak widths change randomly or as a non-trivial function of the atom fraction $X$.

Significant literature exists on the topic of noise reduction in AES. For example, Savitzky and Golay ${ }^{14,17}$ describe a technique of smoothing and obtaining smoothed derivatives using convolution arrays derived from the coefficients of least-squares-fits. Also, the approach of testing noise reducing algorithms by applying them to simulated - thus known - noisy spectra has been employed by others (e. g. Poniku et al., Schwartz and Yoon ${ }^{15,16}$ ) Smoothing (or filtering) the first derivatives of spectra, in particular, addresses the problem that for differentiated peaks only two energy channels determine the signal $S$.

The 9-point derivative applied by the instrument we have employed contributes to reducing the noise in the differentiated spectra, and the software of the instrument offers further options for noise reduction, presumably based on the literature cited above. Under standard acquisition conditions, however, as evident from the fact that the differentiated signal does not properly vanish in regions of vanishing $X$, significant noise remains and leads to artifacts in carbon AFDPs that cannot be tolerated e.g. for quantitative comparison with simulated AFDPs. As shown above, the experimentally observed scatter about the mean value in a region where $X=0$ indicates that the residual noise is random, as assumed by the model described in this work.

The method proposed here has the advantage that the user does not have to interfere at low level with the way the instrument processes the raw data and produces differentiated spectra from it, which is presumably optimized for general application by instrument vendors. The model proposed here may be further developed into a quantitative post-processing routine for background subtraction in SAM composition-depth profiles after differentiation.

\section{CONCLUSIONS}

A quantitative model has been developed to describe the atom-fraction-dependent background in AFDPs (atom-fraction-depth profiles) acquired by SAM (scanning Auger microprobe). The model is based on simplifying assumptions regarding the peak shape, char- acteristics of noise in individual energy channels, and other features of the underlying Auger electron spectra. The model leads to a straight-forward background subtraction strategy based on the resulting "channel statistics" for the derivative of the spectrum.

Supporting this model, the experimentally observed scatter about the mean value in a region in which the actual atom fraction equals zero is consistent with the model. (In the application example, the carbonfree "tail" region of the profile is a zero-atom-fraction region - known not to contain carbon).

For a given detector dispersion (width of the energy channels), the background in the zero-atom-fraction region is determined by the total width of the energy window used in the analysis of the differentiated peak. The background in regions of high at atom fraction, in contrast, is determined by the width of the (nondifferentiated) peak in the Auger electron spectrum and the height of the peak.

\section{CONCLUSIONS}

The following conclusions can be drawn from this analysis:

- For the example of interstitially dissolved carbon diffused into austenitic stainless steel, AFDPs obtained by SAM were studied with regard to noise-induced background.

- The model developed in this work quantitatively explains the background as a result of noise propagation through spectrum differtiation combined with "channel statistics."

- The model is confirmed by being consistent with the experimentally observed scatter about the mean value in the solute-free "tail" region of experimentally acquired AFDPs.

- The model reveals two regimes of background: (i) Background in regions of significant solute levels. In this regime, the background level anticorrelates with the actual local atom fraction and is independent of width of the sampled energy window. (ii) Background in the solute-free "tail" region af AFDPs. In this regime, the background is larger than in any regions of significant solute level. The actual level depends on the total number of channels in the sampled energy window. 
To keep the background in the "tail" region low, the sampled energy window (number of channels) should not be chosen unnecessarily large.

- Owing to the previously mentioned features, it is wrong to subtract the background level observed in the "tail" region everywhere in the AFDP.

- Instead, the model provides a straight-forward quantitative background subtraction strategy that correctly accounts for the dependency of the background level on the local atom fraction. Specifically in regions of significant solute level, background subtraction involves the $\Lambda$ function introduced in this article. This strategy has been verified to work correctly by applying it to simulated noisy AFDPs and restoring the AFDP before noise.
- Complications may arise if the differentiated spectrum involves a multitude of maxima and minima with similar heights/depths or if the peak width is a strong non-trivial function of the atom fraction.

- Future work, including refinement of current assumptions and approximations, may develop this model into a quantitative routine method for background subtraction in SAM compositiondepth profiles.

\section{ACKNOWLEDGEMENTS}

Acknowledgements Removed for Double-Blind Reviewing
${ }^{1}$ L. Meitner, Zeitschrift für Physik 9, 131 (1922).

2 P. Auger, Comptes Rendus de l'Académie des Sciences 177, 169 (1923).

3 D. Briggs and J. T. Grant, Surface Analysis (IM Publications, Manchester, UK, 2003).

4 A. H. Heuer, F. Ernst, H. Kahn, A. Avishai, G. M. Michal, D. J. Pitchure, and R. E. Ricker, Scripta Materialia 56, 1067 (2007).

${ }^{5}$ F. Ernst, A. Avishai, H. Kahn, X. Gu, G. M. Michal, and A. H. Heuer, Metallurgical and Materials Transactions A 40, 1768 (2009).

6 D. Wu, H. Kahn, G. M. Michal, F. Ernst, and A. H. Heuer, Acta Materialia 79, 339 (2014).

7 S. R. Collins, P. C. Williams, S. V. Marx, A. H. Heuer, F. Ernst, and H. Kahn, in ASM Handbook: Vol. 4D, Heat Treating of Irons and Steels, edited by J. Dossett and G. Totten (ASM International, Materials Park, Ohio, 2014), vol. 4, pp. 451-460.

8 Y. Cao, F. Ernst, and G. Michal, Acta Materialia 51, 4171 (2004).

9 J. Crank, The Mathematics of Diffusion (Clarendon Press,
Oxford, 1956).

10 S. Hofmann, Auger-and X-Ray Photoelectron Spectroscopy in Materials Science, Springer Series in Surface Sciences (Springer, Berlin, 2013).

11 P. W. Palmberg, Annalen der Chemie 45, 549A (1973).

12 I. Wolfram Research, Mathematica (Wolfram Research, Inc., Champaign, Illinois, 2016), version 11.0 ed.

13 L. Davis, N. C. McDonald, P. W. Palmberg, G. E. Riach, and R. E. Weber, Handbook of Auger Electron Spectroscopy (Physical Electronics Industries, Eden Prairie, MN, USA, 1976).

14 A. Savitzky and M. J. E. Golay, Analytical Chemistry pp. 1627-1639 (1964).

15 B. Poniku, I. Beli, and M. Jenko, Materials and Technology 49, 435 (2015).

16 S. Schwarz and H. Yoon, Applied Surface Science 31, 189 (1988).

17 I. Gilmore and M. Seah, Applied Surface Science 93, 273 (1996). 